\title{
Uveítis secundaria a Borreliosis de Lyme en México
}

\section{Uveitis associated with Lyme disease in Mexico}

\author{
Alejandro Rodríguez-García ${ }^{1 *}$, Isidora Arroyo-Garza² y Beatriz E. Patiño-Ramirez ${ }^{2}$ \\ ${ }^{1}$ Servicio de Inmunología Ocular y Uveítis, Instituto de Oftalmología y Ciencias Visuales; ${ }^{2}$ Programa Multicéntrico de Residencia en Oftalmología, \\ Tecnológico de Monterrey, Escuela de Medicina y Ciencias de la Salud. Nuevo León, México
}

\section{Resumen}

Objetivo: La borreliosis de Lyme es una infección multisistémica rara, y al igual que ocurre en otros países, en México rara vez se considera en el diagnóstico diferencial de ciertas formas de uveítis debido al desconocimiento de los factores de riesgo de contagio por Borrelia burgdorferi. El presente reporte tiene como objetivo revisar dichos factores de riesgo, las características clínicas de la afección ocular, así como su sospecha en pacientes susceptibles. Observaciones: Paciente femenina, 4 años, con ojo rojo y visión borrosa bilateral de 6 semanas de evolución. Mialgias-artralgias, erupción cutánea y cuadro respiratorio las 10 semanas previas. Tratada como panuveítis bilateral con esteroides tópicos y sistémicos por 20 días sin mejoría. Agudeza visual 20/200, células 3+ en cámara anterior y vitreítis difusa 2+ en ambos ojos. Pérdida de bordes del nervio óptico derecho; exudados perivasculares e infiltrados coriorretinianos color blanco-cremoso en la periferia en ambos ojos. Se diagnosticó coriorretinitis multifocal, papilitis y vasculitis retiniana con serología positiva para B. burgdorferi. Tratada con amoxicilina $250 \mathrm{mg}$ cada $8 \mathrm{~h}$ por vía oral por 3 semanas con resolución del cuadro inflamatorio. Conclusiones: El diagnóstico de borreliosis de Lyme en etapas tempranas es difícil debido a los signos inespecíficos y la seroconversión inconstante. La uveítis por Borrelia burgdorferi debe considerarse en todo paciente bajo riesgo que presente manifestaciones neurooftalmológicas asociadas a uveítis bilateral crónica refractaria a tratamiento con esteroides.

Palabras clave: Borreliosis ocular. Lyme. Borrelia burgdorferi. Uveítis. Coriorretinits. Papilitis.

\section{Abstract}

Objective: Lyme disease is a rare multi-systemic infection and like in many other countries, in Mexico it is rarely considered in the differential diagnosis of certain forms of uveitis due to lack of knowledge about the risk factors of Borrelia burgdorferi infection. This report aims to review such risk factors, the clinical characteristics of ocular involvement, as well as the keys to suspect the disease in susceptible patients. Observations: A 4-year-old female with a 6-week history of bilateral red eye and blurred vision. The patient referred muscular and joints pain, skin rash, and respiratory symptoms 10 weeks before. She was previously treated for bilateral panuveitis with topical and systemic steroids during 20 days without improvement. Visual acuity was 20/200, with $3+$ cells in the anterior chamber, and 2+ diffuse vitritis. Blurred right disk margins, perivascular sheathing, and multiple chorioretinal white-cream-colored infiltrates in the peripheral retina of both eyes. Multifocal chorioretinitis, papillitis, and vasculitis were diagnosed with a positive serology for B. burgdorferi. Treatment consisted in $250 \mathrm{mg}$ of oral

\section{Correspondencia:}

*Alejandro Rodríguez García

Av. Batallón de San Patricio, 112

Col. Real de San Agustín

C.P. 66278, San Pedro Garza García, $\quad$ Fecha de recepción: 09-03-2018

N.L., México

Fecha de aceptación: 01-06-2018

Disponible en internet: 27-07-2018

E-mail: immuneye@gmail.com

DOI: 10.24875/RMO.M18000039

Rev Mex Oftalmol. 2020;94(1):39-45 www.rmo.com.mx

0187-4519/@ 2018 Sociedad Mexicana de Oftalmología. Publicado por Permanyer. Éste es un artículo open access bajo la licencia CC BY-NC-ND (http://creativecommons.org/licenses/by-nc-nd/4.0/). 
amoxicillin every $8 \mathrm{~h}$ for 3 weeks, with complete resolution of inflammation. Conclusions: The diagnosis of Lyme disease at early stages is difficult due to its unspecific signs and inconsistent serology. Uveitis due to B. burgdorferi must be considered in every patient at risk who presents with neuro-ophthalmologic manifestations associated with chronic bilateral uveitis refractory to steroid therapy.

Key words: Ocular borreliosis. Lyme. Borrelia burgdorferi. Uveitis. Chorioretinitis. Neuroretinitis.

\section{Introducción}

La borreliosis de Lyme es una enfermedad infecciosa emergente multisistémica causada por la espiroqueta Borrelia burgdorferi, que se transmite por la mordedura de garrapatas del género lxodes, vector de la enfermedad $^{1}$. El Ixodes scapularis, vector más frecuente en EE.UU. y presente en el estado de Texas, también se ha encontrado en zonas de Coahuila ${ }^{2}$. Además, se han identificado Ixodes en la Baja California, la península de Yucatán, el golfo de México y la zona del noreste de la República Mexicana ${ }^{3-5}$. El riesgo de contagio está directamente relacionado con la prevalencia de garrapatas y de reservorios infectados, así como la exposición a zonas endémicas ${ }^{6}$.

En México, los primeros casos de eritema crónico migratorio asociado a borreliosis de Lyme fueron reportados en Sinaloa y Monterrey en 19917,8. Ocho años después, Gordillo, et al reportaron una prevalencia de infección por $B$. burgdorferi del $1.1 \%$ en la población general de México, mientras que la prevalencia del venado cola blanca del noreste de nuestro país fue del $3 \%{ }^{4}$. Los venados son hospederos finales de los Ixodes adultos y su función primaria es mantener al vector. Otro estudio detectó Borrelia spp en el $16 \%$ de los perros residentes del área metropolitana de Monterrey ${ }^{10}$. Para el año 2003 se había reportado una seroprevalencia de infección por B. burgdorferi del $3.4 \%$ en la ciudad de México y del $6.3 \%$ en la población general del noreste del país, incluyendo los estados de Nuevo León, Coahuila y Tamaulipas, este último era el de mayor seroprevalencia ${ }^{5}$. En 2007 se reportaron los primeros casos confirmados de enfermedad cutánea y neurológica procedentes del valle de México y de Quintana Roo ${ }^{11}$.

La tercera parte de los casos de borreliosis de Lyme se presentan en menores de 18 años, y la edad de mayor riesgo es entre los 5 y los 14 años de edad ${ }^{12}$. En México, desde 1999 se ha mantenido una vigilancia epidemiológica para identificar casos de borreliosis de Lyme en la infancia ${ }^{13}$. Hasta el año 2010 se habían estudiado 72 niños, con una edad promedio de 8.5 años, de los cuales, el $55 \%$ presentaron manifestaciones cutáneas, el $27 \%$, neurológicas, y $18 \%$ presentaron artritis. El $31.9 \%$ dieron positivo al estudio por ensayo enzimático inmunoabsorbente (ELISA), y se confirmó enfermedad de Lyme en $28 \%$ de ellos por Western blot ${ }^{13}$. El $75 \%$ de estos niños presentaron manifestaciones tempranas de la enfermedad, lo que explica el bajo índice de seropositividad, mientras que el $25 \%$ restante presentaron manifestaciones tardías, pero en ninguno de estos casos se identificaron manifestaciones oculares ${ }^{13}$.

La borreliosis de Lyme puede presentar diversas manifestaciones oftalmológicas que se agrupan en: desórdenes neurooftalmológicos, inflamación ocular externa, inflamación intraocular y desórdenes vasculares de la retina ${ }^{14}$. La borreliosis ocular de Lyme sigue siendo una enfermedad subdiagnosticada en parte debido al desconocimiento de las manifestaciones oftalmológicas y su falta de inclusión en el diagnóstico diferencial de diversos trastornos inflamatorios oculares, pero también por la dificultad de obtener un diagnóstico serológico definitivo ${ }^{15,16}$. En el presente reporte presentamos el primer caso de uveítis secundaria a borreliosis de Lyme descrito en México.

\section{Presentación del caso}

Paciente femenina de 4 años de edad, originaria de Monclova Coahuila, que fue referida al Servicio de Inmunología y Uveítis de nuestra institución para una segunda opinión por ojo rojo, fotofobia extrema y visión borrosa bilateral de 6 semanas de evolución. La paciente pertenece a una clase socioeconómica media-baja, que tiene como antecedente de importancia haber vivido en la zona semirrural de la ciudad de Longview al noreste de Texas, zona geográfica considerada área endémica de enfermedad de Lyme por el Centro de Control de Enfermedades (CDC) de Atlanta, Georgia, EE.UU., y también en el área suburbana de Monclova, Coahuila, en el noreste de México.

La paciente había sido tratada por panuveítis bilateral con esteroides tópicos y sistémicos durante 20 días, sin mejoría, pero con deterioro de su estado visual e inflamatorio ocular. Al interrogatorio indirecto (padres) por aparatos y sistemas refería mialgias y artralgias en los miembros inferiores y un cuadro similar a infección 
de las vías respiratorias superiores, manifestado como fiebre, ataque al estado general, rinorrea y erupción cutánea de 8 días de duración, cuadro que se presentó 10 semanas antes de su consulta. Al examen físico, la paciente no presentaba manifestaciones cutáneas, neurológicas o reumáticas evidentes asociadas a enfermedad de Lyme. El examen oftalmológico inicial reveló una agudeza visual mejor corregida de 20/200, presentando miopía (-1.25 dioptrías) y astigmatismo leve ( -0.50 dioptrías) en ambos ojos. Al examen externo se encontró inyección ciliar 1+y a la biomicroscopia se observaron precipitados retroqueráticos endoteliales finos de distribución media-inferior y celularidad de $3+$ en la cámara anterior. El iris no presentaba alteraciones patológicas y el cristalino se encontró claro. A la fondoscopia se apreció una vitreítis difusa de 2+, pérdida sutil de los bordes del nervio óptico derecho (Fig. 1), exudados perivasculares segmentarios periféricos de predominancia venular, así como múltiples infiltrados inflamatorios coriorretinianos redondos de aproximadamente $50 \mathrm{~mm}$ de diámetro, de color blanco-cremoso en la periferia (Fig. 2). Se diagnosticó coriorretinitis multifocal, papilitis y vasculitis retiniana bilateral. En el abordaje inicial se consideraron dentro del diagnóstico diferencial, además de la artritis idiopática juvenil y el lupus eritematoso sistémico, etiologías infecciosas, como: sífilis, tuberculosis, toxoplasmosis multifocal atípica, bartonelosis y borreliosis de Lyme. Se solicitaron los siguientes estudios: biometría hemática, perfil bioquímico, proteína C-reactiva, velocidad de sedimentación eritrocítica, examen general de orina, anticuerpos antinucleares, determinación serológica de fracciones del complemento $\mathrm{C} 3, \mathrm{C} 4$ y $\mathrm{CH} 50$, prueba cutánea de tuberculina (PPD), determinación de anticuerpos fluorinados contra el Treponema Pallidum (FTA-Abs), prueba serológica para la sífilis (VDRL), anticuerpos IgG e IgM contra Toxoplasma gondii, Bartonella henselae y $B$. burgdorferi (ELISA). Los resultados de las pruebas serológicas fueron negativos, excepto por el análisis serológico diferenciado («2-tiered») para borreliosis de Lyme, el cual dio positivo para anticuerpos IgM (resultado: 1.21; referencia: positivo > 1.09) e IgG (resultado: 1.35; referencia: positivo > 1.09) por ELISA, confirmado por Western-inmunoblot. Este último arrojó positivo para las bandas IgM p41Kd (flagelina), IgM p23Kd (Osp C) e IgG p41Kd, específicas de B. burgdorferi (LabCorp - Laboratory Corporation of America, Burlington, NC. EE.UU.; avalado por el CDC de Atlanta, GA.)

Basado en los antecedentes de habitar en una zona suburbana de Coahuila, el viaje y estancia en una zona

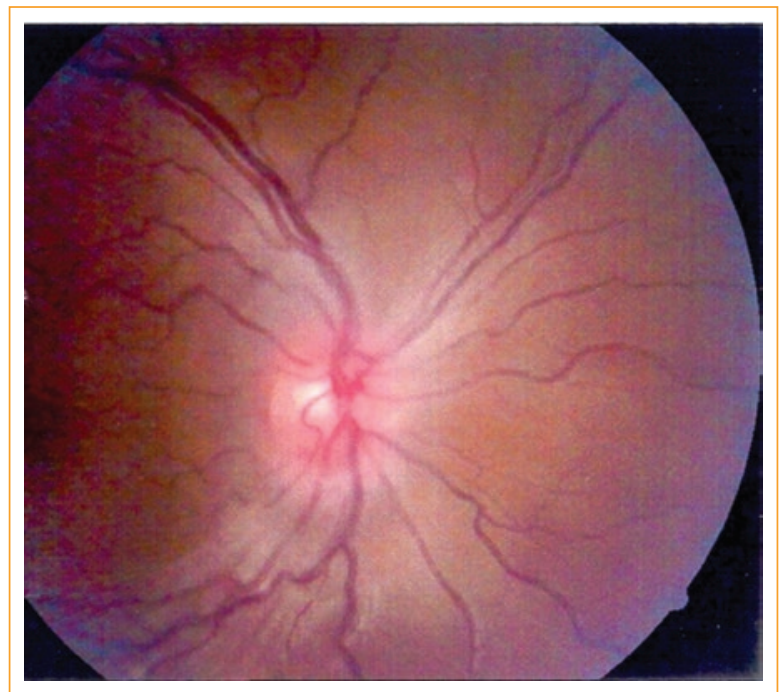

Figura 1. Nervio óptico derecho con márgenes no definidos, ligera hiperemia y congestión vascular.

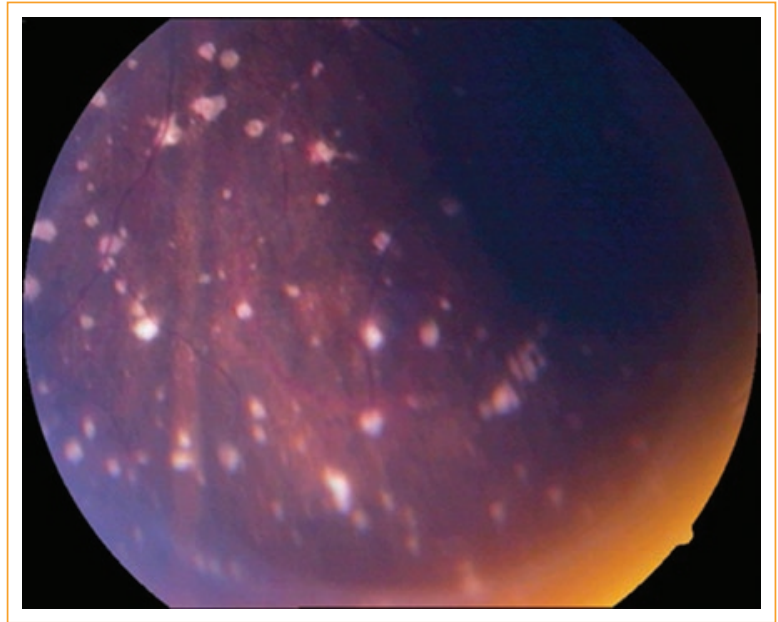

Figura 2. Lesiones multifocales, blanco-cremosas, profundas en la retina y coroides en el cuadrante inferior-temporal del ojo derecho.

semirrural al noreste de Texas, la presencia de un cuadro respiratorio agudo con ataque al estado general, mialgias, artralgias, fiebre y erupción cutánea de más de una semana de duración, además de la ausencia de respuesta a esteroides tópicos y sistémicos previos, así como los hallazgos fondoscópicos y la confirmación serológica de $B$. burgdorferi, se llegó al diagnóstico de uveítis secundaria a borreliosis de Lyme. Se inició tratamiento tópico a base de acetato de fluorometolona al $0.1 \%$ cada $4 \mathrm{~h}$ y amoxicilina $250 \mathrm{mg}$ cada $8 \mathrm{~h}$ (dosis ponderal: $50 \mathrm{mg} / \mathrm{kg} / \mathrm{día}$ ) vía oral durante tres semanas. 
Al término del tratamiento se observó ausencia de células inflamatorias en la cámara anterior y la cavidad vítrea; los bordes del nervio óptico estaban bien definidos (Figs. 3 A y 3 B); había ausencia de vasculitis retiniana y en la retina periférica las lesiones coriorretinianas estaban atróficas con apariencia «en sacabocado" (Figs. 4 A y 4 B). La agudeza visual mejor corregida era de 20/50 en ojo derecho y 20/40 en ojo izquierdo. En su última visita de seguimiento, 4 meses postratamiento, la paciente se encontró asintomática, sin datos de actividad inflamatoria intraocular y con una agudeza visual estable. Una nueva serología diferenciada para borreliosis de Lyme arrojó resultados negativos para anticuerpos por ELISA y ausencia de bandas específicas para $B$. burgdorferi por Western-inmunoblot. La paciente acudió 2 años después a revisión por miopía y astigmatismo, se encontraba asintomática y sin datos de uveítis activa.

\section{Discusión}

La borreliosis de Lyme es la enfermedad transmitida por vectores más frecuente en EE.UU. y algunos países de Europa ${ }^{17,18}$. A pesar de ser reportada hasta 6 a 12 veces menos de lo estimado, aproximadamente 30,000 casos son reportados al CDC de EE.UU. cada año ${ }^{18,19}$. El contexto epidemiológico es muy importante para el diagnóstico temprano de la enfermedad de Lyme. La mordedura por garrapata ocurre con mayor probabilidad en personas que pasan tiempo en hábitats arbolados o con arbustos y pastizales de lugares endémicos, especialmente entre mayo y noviembre ${ }^{19}$. En el presente caso, los antecedentes de haber vivido en una zona endémica del noreste de Texas, en EE.UU. ${ }^{20}$, y de vivir en la zona noreste de México, lugar donde se ha reportado la mayor seroprevalencia de infección por $B$. burgdorferi ${ }^{5}$, así como la de mayor presencia de la garrapata Ixodes scapularis $^{2,21}$ y de su hospedero natural, el venado cola blanca ${ }^{4}$, promovieron la consideración de la borreliosis de Lyme como parte del diagnóstico diferencial.

Las manifestaciones clínicas de la enfermedad de Lyme han sido divididas en tres etapas: localizada, diseminada y persistente ${ }^{1}$. Las primeras dos etapas son parte de la infección temprana por $B$. burgdorferi, mientas que la enfermedad persistente es considerada como una infección tardía ${ }^{22}$. La tabla 1 muestra las principales manifestaciones multisistémicas y oftalmológicas, así como la duración y metodología diagnóstica empleada en las diversas etapas de la enfermedad.

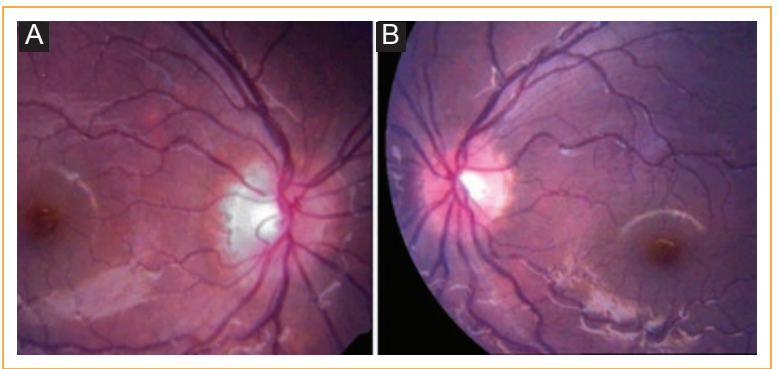

Figura 3. Nervios ópticos derecho (A) e izquierdo (B), que muestran bordes bien definidos y sin congestión vascular.

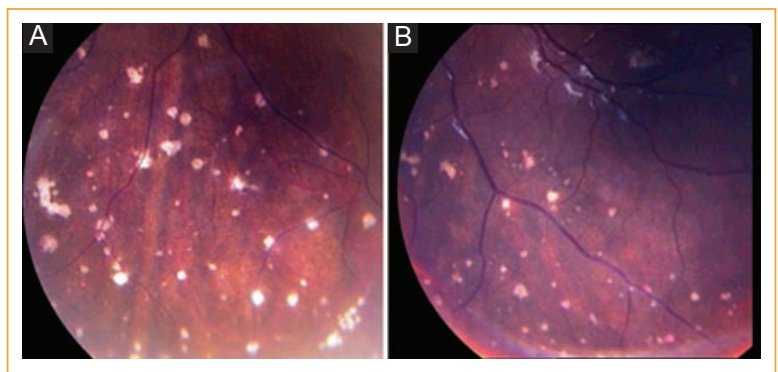

Figura 4. Retina periférica del ojo derecho (A) y del ojo izquierdo (B) que muestra múltiples lesiones atróficas coriorretinianas de distribución difusa y vítreo claro.

Se ha calculado que las manifestaciones oculares ocurren en tan solo el 1\% de los casos, y pueden ocurrir en cualquiera de las tres etapas de la enfermedad $^{14,22}$. Durante la primera etapa, caracterizada por la aparición del eritema migratorio y un cuadro febril indiferenciado, puede observarse conjuntivitis, ojo rojo, fotofobia y lagrimeo ${ }^{23,24}$. La conjuntivitis es la manifestación más común, reportada en hasta el $11 \%$ de los casos y casi siempre se presenta en las primeras semanas de la infección ${ }^{23}$. En la segunda etapa, dominada por síntomas neurológicos y musculoesqueléticos, puede observarse diplopía, parálisis facial, oftalmoplegia internuclear, visión borrosa y dolor ocular, así como queratitis, epiescleritis, uveítis anterior, coriorretinitis multifocal, papilitis, panoftalmitis, obstrucciones venosas de retina, desprendimiento exudativo de retina y miositis orbitaria ${ }^{14,24,25}$. En la tercera etapa, caracterizada por síntomas reumatológicos (artritis) y neurológicos, suelen ocurrir todas las manifestaciones oculares descritas en la segunda etapa, pero sobre todo las más severas, como la inflamación intraocu$\operatorname{lar}^{12,16,24,26}$. La uveítis intermedia es la forma más 
Tabla 1. Características clínicas sistémicas y oculares en las diversas etapas de la borreliosis de Lyme

\begin{tabular}{|c|c|c|c|}
\hline Etapa & Manifestaciones multisistémicas & $\begin{array}{l}\text { Manifestaciones } \\
\text { oftalmológicas }\end{array}$ & $\begin{array}{l}\text { Tíempo de aparición y método } \\
\text { diagnóstico }\end{array}$ \\
\hline $\begin{array}{l}\text { Primera: Temprana } \\
\text { localizada }\end{array}$ & $\begin{array}{l}\text { Eritema migratorio ( } 66 \% \text { casos), inicia } \\
7 \text { días posmordedura de garrapata y } \\
\text { persiste por } 2-3 \text { semanas. Enfermedad } \\
\text { febril indiferenciada ( } 50 \% \text { casos), inicia } \\
\text { 1-7 días posmordedura. Fiebre, } \\
\text { escalofríos, malestar general, fatiga, } \\
\text { mialgias, artralgias y cefalea. }\end{array}$ & $\begin{array}{l}\text { Conjuntivitis, ojo rojo, } \\
\text { fotofobia y lagrimeo. }\end{array}$ & $\begin{array}{l}\text { Inician 1-30 días posinfección. } \\
\text { Diagnóstico clínico. No se } \\
\text { recomiendan pruebas } \\
\text { serológicas (falsos negativos). }\end{array}$ \\
\hline $\begin{array}{l}\text { Segunda: Temprana } \\
\text { diseminada }\end{array}$ & $\begin{array}{l}\text { Neurológicas ( } 5-20 \% \text { casos), parálisis } \\
\text { facial, más común, meningitis y } \\
\text { radiculopatía (tríada de Lyme), así } \\
\text { como encefalopatía y } \\
\text { musculo-esqueléticas: poliartritis } \\
\text { migratoria que evoluciona a proceso } \\
\text { monoarticular (rodilla, tobillo y } \\
\text { muñeca). }\end{array}$ & $\begin{array}{l}\text { Diplopía, oftalmoplegia } \\
\text { internuclear, parálisis } \\
\text { facial, papiledema, visión } \\
\text { borrosa, dolor, queratitis, } \\
\text { epi y escleritis, } \\
\text { uveítis (coriorretinitis } \\
\text { multifocal), papilitis, } \\
\text { panoftalmitis. }\end{array}$ & $\begin{array}{l}\text { Artritis, inicia } 3-10 \text { semanas. y } \\
\text { meningitis, } 2-10 \text { semanas posinfección. } \\
\text { Anticuerpos IgM e IgG contra } \\
B . \text { Burgdorferi (ELISA), confirmado por } \\
\text { Western-blot. PCR (disponibilidad } \\
\text { limitada) }\end{array}$ \\
\hline $\begin{array}{l}\text { Tercera: Tardía o } \\
\text { crónica } \\
\text { persistente }\end{array}$ & $\begin{array}{l}\text { Reumáticas: artritis de rodilla ( } 90 \% \\
\text { casos) y neurológicas: encefalopatía } \\
\text { subaguda, encefalomielitis crónica } \\
\text { progresiva, neuropatía axonal y } \\
\text { mielitis ( } 50 \% \text { casos). } \\
\text { Acrodermatitis crónica atropicana en } \\
\text { dorso de manos, pies, rodillas y } \\
\text { codos (europeos). }\end{array}$ & $\begin{array}{l}\text { Uveítis anterior, } \\
\text { intermedia, posterior, } \\
\text { panuveítis, vasculitis } \\
\text { retiniana, miositis } \\
\text { orbitaria, } \\
\text { desprendimiento } \\
\text { exudativo de retina, } \\
\text { obstrucción de vena } \\
\text { central y de rama. }\end{array}$ & $\begin{array}{l}\text { Inician meses a años posinfección } \\
\text { inicial. Anticuerpos IgG contra } \\
\text { B. Burgdorferi (ELISA), confirmado por } \\
\text { Western-blot. PCR (disponibilidad } \\
\text { limitada) }\end{array}$ \\
\hline
\end{tabular}

ELISA: ensayo enzimático inmuno-absorbente; PCR: reacción en cadena de polimerasas.

común, pero también se observa la coriorretinitis multifocal como ocurrió en el presente caso y la vasculitis retiniana ${ }^{14}$. No se ha establecido si los pacientes en etapa tardía representan una falla al tratamiento antibiótico o persistencia de la borrelia, una nueva infección con otro patógeno transmitido por garrapatas o tal vez un fenómeno de autoinmunidad ${ }^{26}$.

En el presente reporte, todo hace suponer que la paciente se encontraba en la segunda etapa por el tiempo transcurrido desde el cuadro respiratorio y febril inespecífico hasta la aparición de los síntomas oculares. Aparte del resultado serológico positivo y el tipo de manifestación ocular: coriorretinitis multifocal, vasculitis retiniana y papilitis, que son manifestaciones clínicas concordantes con esta etapa, la paciente no presentó síntomas reumáticos, ni neurológicos.

El eritema migratorio es el indicador diagnóstico clínico de la borreliosis de Lyme; sin embargo, hasta en el $20 \%$ de los casos no se observa erupción cutánea ${ }^{27}$. En áreas endémicas, los pacientes con eritema migratorio y reciente exposición a mordedura por garrapata no requieren de estudios serológicos para el diagnóstico ${ }^{19,27}$. En estas primeras etapas de la enfermedad, la posibilidad de resultados serológicos falsos negativos es alta y cuando dichas pruebas resultan positivas, no logran distinguir entre una infección activa o pasada ${ }^{28}$. En este mismo sentido, la sensibilidad del análisis serológico diferenciado («2-tiered») para detectar anticuerpos IgM e IgG contra $B$. burgdorferi en el suero de pacientes cuando la respuesta inmune humoral se está desarrollando es tan solo del $30-40 \%{ }^{28,29}$. Por tanto, el diagnóstico de borreliosis ocular de Lyme en etapas tempranas es difícil, pues está basado en hallazgos clínicos inespecíficos relacionados con inflamación de la superficie ocular, inflamación intraocular o alteraciones neurooftalmológicas de pacientes en alto riesgo de contagio $^{30}$. Para la enfermedad de Lyme diseminada, la sensibilidad de las pruebas serológicas se incrementa al $70-100 \%$, mientras que la especificidad es alta $(95 \%)$ durante todas las etapas de la enfermedad ${ }^{15}$. Otras pruebas diagnósticas son las directas, específicamente el cultivo y la reacción en cadena de la polimerasa (PCR). El cultivo no está disponible para su realización en forma rutinaria, técnicamente es difícil de realizar y se caracteriza por su baja sensibilidad diagnóstica. Por otra parte, la PCR solo se realiza en forma 
ordinaria en líquido sinovial de pacientes con artritis sospechosos de padecer la enfermedad, y existen reportes aislados de su utilidad diagnóstica en tejidos oculares, como conjuntiva, humor acuoso o vítreo ${ }^{31,32}$.

Entonces, el diagnóstico de la uveítis secundaria a borreliosis de Lyme está basado en los antecedentes de exposición a mordedura por garrapata o ambientes de riesgo; en un análisis serológico diferenciado positivo durante las etapas diseminada y persistente; en la resistencia al tratamiento con esteroides, y en la respuesta favorable a la antibióticoterapia, así como en la exclusión de otros diagnósticos probables ${ }^{33,34}$. En este caso, la paciente tenía antecedentes epidemiológicos de riesgo para desarrollar la borreliosis de Lyme, no había mostrado mejoría al tratamiento con esteroides y la serología para otras causas de uveítis fue negativa. Además, presentó un resultado francamente positivo y confirmatorio de infección con $B$. burgdorferi en la serología diferenciada. Al respecto, la paciente presentó bandas en la electroforesis proteica de p23Kd y p41Kd para IgM y de p41Kd para IgG contra B. burgdorferi, las cuales son típicas en la enfermedad de Lyme, que se tornaron negativas después del cuarto mes postratamiento ${ }^{27,29}$. Durante el curso clínico de la borreliosis de Lyme se observa una seroconversión por ELISA y Western-blot, que cambia de positiva a negativa y viceversa sin seguir un patrón regular ${ }^{35}$.

El diagnóstico diferencial de la borreliosis ocular de Lyme debe incluir cualquiera de las causas probables de las manifestaciones neurooftalmológicas e inflamatorias oculares observadas en estos casos, incluyendo la conjuntivitis viral, infecciones por virus herpes simple y varicela zoster, fiebre de las Montañas Rocosas, síndrome de Horner, sarcoidosis, parálisis facial y troclear, neuritis óptica, sarcoidosis, sífilis, entre otras ${ }^{22,24,36}$.

En cuanto al manejo, la conjuntivitis de la primera etapa usualmente no requiere tratamiento, al igual que la parálisis facial presente en la segunda etapa, que suele ser autolimitada, pero las complicaciones de la queratitis por exposición requieren terapia de soporte. La epiescleritis y queratitis suelen tratarse con un curso corto de esteroides tópicos ${ }^{25}$. Por otra parte, en el caso de enfermedad neurooftalmológica con afectación del nervio óptico o uveítis intermedia, posterior y panuveítis, el uso de esteroides sistémicos debe acompañarse de antibiótico-terapia. Para ello, se emplean 2 a 3 semanas de penicilina-G, doxiciclina, cefuroxima, cefotaxima o ceftriaxona en la mayoría de los $\operatorname{casos}^{26}$. En la infancia, el antibiótico oral de primera elección es la amoxicilina (50 mg/kg/día hasta una dosis máxima de $500 \mathrm{mg} / \mathrm{día}$ ), es por ello que en este caso decidimos emplear dicho antibiótico, aunque también se puede emplear penicilina- $V$ sódica y ceftriaxona ${ }^{37}$. Una revisión sistemática de la antibiótico-terapia en niños con borreliosis de Lyme no encontró diferencias en la eficacia terapéutica entre los diferentes antibióticos ${ }^{37}$. Las recurrencias de la borreliosis de Lyme, sobre todo la artritis en tercera etapa se trata con esteroides ${ }^{26}$.

Nuestra paciente tenía 4 años de edad, se encontraba en la segunda etapa y había recibido esteroides sistémicos durante 3 semanas sin respuesta. Por estos motivos se decidió suspenderlos. En conclusión, la uveítis secundaria a borreliosis de Lyme es una entidad rara y probablemente subdiagnosticada en nuestro país. La escasa información epidemiológica y la falta de un escrutinio serológico a pacientes sospechosos de contraer la enfermedad contribuyen al bajo diagnóstico. En el diagnóstico de cualquier panuveítis bilateral se debe incluir un interrogatorio exhaustivo de aparatos y sistemas que incluya los antecedentes de modo y lugar de vida (zona geográfica), especialmente de hábitats de alto riesgo para el contagio con $B$. burgdorferi. En nuestro país se deben considerar dichos antecedentes, especialmente en pacientes provenientes de la zona noreste y su franja fronteriza con el estado de Texas, EE.UU. En cualquier paciente sospechoso o en riesgo que presente manifestaciones neurooftalmológicas asociadas a uveítis bilateral en quien se hayan descartado otros procesos infecciosos e inmunes frecuentes y que no respondan a esteroides, se debe tomar en cuenta a la borreliosis de Lyme dentro del diagnóstico diferencial.

\section{Responsabilidades éticas}

Protección de personas y animales. Los autores declaran que para esta investigación no se han realizado experimentos en seres humanos ni en animales.

Confidencialidad de los datos. Los autores declaran que han seguido los protocolos de su centro de trabajo sobre la publicación de datos de pacientes.

Derecho a la privacidad y consentimiento informado. Los autores declaran que en este artículo no aparecen datos de pacientes.

\section{Conflicto de intereses}

Ninguno que reportar.

\section{Bibliografía}

1. Pfister HW, Wilske B, Weber K. Lyme borreliosis: basic science and clinical aspects. Lancet. 1994;343:1013-6.

2. Keirans JE, Hutcheson HJ, Durden LA, Klompen JSH. Ixodes (Ixodes) scapularis (Acari: Ixodidae): redescription of all active stages, distribution, 
hosts, geographical variation, and medical and veterinary importance. J Med Entom. 1996;33:297-318.

3. Kohls GM, Clifford CM. Three new species of Ixodes from Mexico and description of the male of $I$. auritulus auritulus Neumann, I. conepati Cooley and Kohls, and I. Iasallei Mendez and Ortiz (Acarina: Ixodidae). J Parasitol. 1966;52:810-20.

4. Martinez A, Salinas A, Martinez F, Cantu A, Miller DK. Serosurvey for selected disease agents in white-tailed deer from Mexico. J Wild Life Dis. 1999;35:799-803.

5. Gordillo-Pérez G, Torres J, Solórzano-Santos F, Garduño-Bautista V, Tapia-Conyer R, Muñoz HO. Estudio seroepidemiológico de borreliosis de Lyme en la ciudad de México y el noreste de la república mexicana. Salud Publica Mexico. 2003;45:351-5

6. Steere AC, Malawista SE. Cases of Lyme disease in the United States: Locations correlated with distribution of Ixodes dammini. Ann Intern Med. 1979;91:730-3.

7. Vargas MH. Lyme disease in Mexico City. Salud Publica Mexico. 1993;35:435-6.

8. Arroyave CM, González RT. Enfermedad de Lyme: Informe de dos casos. Bol Med Hosp Infant Mex. 1994;51:117-21.

9. Gordillo-Pérez G, Solórzano-Santos F, Torres L, Cedillo RR, Tapia CR, Muñoz HO. Serological evidence of Lyme disease in Mexico. Arch Med Res. 1999;30:64-8.

10. Salinas-Meléndez JA, Avalos-Ramírez R, Riojas-Valdez VM, Martínez-Muñoz A. Serological survey of canine borreliosis. Rev Latinoam Microbiol. 1999;41:1-3.

11. Gordillo-Pérez G, Torres J, Solórzano-Santos F, de Martino S, Lipsker D, Velazquez E, et al. Borrelia burgdorferi infection and cutaneous Lyme disease, Mexico. Emerg Infect Dis. 2007;13:1556-8.

12. Huppertz HI, Münchmeier D, Lieb W. Ocular manifestations in children and adolescents with Lyme arthritis. Br J Ophthalmol. 1999;83:1149-52.

13. Gordillo-Pérez MG, Solórzano-Santos F. Enfermedad de Lyme. Experiencia en niños mexicanos. Bol Med Hosp Infant Mexico. 2010;67: 164-76.

14. Mikkilä HO, Seppälä IJ, Viljanen MK, Peltomaa MP, Karma A. The expanding clinical spectrum of ocular lyme borreliosis. Ophthalmology. 2000;107:581-7.

15. Moore A, Nelson C, Molins C, Mead P, Schriefer M. Current guidelines, common clinical pitfalls, and future directions for laboratory diagnosis of Lyme disease, United States. Emerging Infect Dis. 2016;22:1-9.

16. Rodriguez-Garcia A, Vidaurri-Leal JS. Ocular manifestations of systemic granulomatous diseases. Current Opinion Ophthalmol. 1991;2:181-89.

17. van den Wijngaard CC, Hofhuis A, Simões M, Rood E, van Pelt W, Zeller $\mathrm{H}$, et al. Surveillance perspective on Lyme borreliosis across the European Union and european econonomic area. Eurosurveillance.com 2017;22:1-9.

18. Centers for Disease Control and Prevention. Lyme disease data. CDC. Disponible en: http://www.cdc.gov/lyme/stats/index.html?s_cid=cs_281. September 24, 2015; Último acceso: 14 agosto, 2017.

19. Mead PS. Epidemiology of Lyme disease. Infect Dis Clin North America. 2015;29:187-210.
20. Dandashi JA, Nizamutdinov D. Texas occurrence of Lyme disease and its neurological manifestations. J Neuroinfect Dis. 2016;7:1-5.

21. Skinner-Taylor CM, Flores-González MDS, Valerio E, Antonio J, Salinas-Meléndez JA, Salinas-Palacios, et al. Evidencia de la enfermedad de Lyme en una población de alto riesgo del noreste de México. Medicina Universitaria. 2007;9:105-11.

22. Karma A, Seppälä I, Mikkilä H, Kaakkola S, Viljanen M, Tarkkanen A Diagnosis and clinical characteristics of ocular Lyme borreliosis. Am J Ophthalmol. 2014;119:127-135.

23. Mombaerts I, Maudgal PC, Knockaert D. Bilateral follicular conjunctivitis as a manifestation of Lyme disease. Am J Ophthalmol. 1991;112:96-7.

24. Raja H, Starr MR, Bakri SJ. Ocular manifestations of tick-borne diseases. Surv Ophthalmol. 2016;61:726-44.

25. Flach AJ, Lavoie PE. Episcleritis, conjunctivitis, and keratitis as ocular manifestations of Lyme disease. Ophthalmology. 1990;97:973-5.

26. Meyerhoff JO, Steele RW, Zaidman GW. Lyme Disease. Update June 29, 2017. In emedicine.medscape.com/article/330178. Último acceso: 2 septiembre, 2017.

27. Centers for Disease Control and Prevention (CDC) Recommendations for test performance and interpretation from the Second National Conference on Serologic Diagnosis of Lyme Disease. MMWR. 1995;44:590-1.

28. Marques AR. Laboratory diagnosis of Lyme disease: Advances and challenges. Infect Dis Clin North America. 2015;29:295-307.

29. Branda JA, Strle K, Nigrovic LE, Lantos PM, Lepore TJ, Damle NS, et al. Evaluation of modified 2-tiered serodiagnostic testing algorithms for early Lyme disease. Clin Infect Dis. 2017;64:1074-80.

30. Kazi H, de Groot-Mijnes JDF, Dam-van Loon ten NH, Norel JO-V, Oosterheert $\mathrm{JJ}$, de Boer $\mathrm{JH}$. No value for routine serologic screening for Borrelia burgdorferi in patients with uveitis in the Netherlands. Am J Ophthalmol. 2016;166:189-93.

31. Marques AR. Laboratory diagnosis of Lyme disease - Advances and challenges. Infect Dis Clin North Am. 2015;29:295-307.

32. Mikkilä H, Karma A, Viljanen M, Seppälä I. The laboratory diagnosis of ocular Lyme borreliosis. Graefe's Arch Clin Exp Ophthalmol. 1999; 237:225-30.

33. Bernard A, Kodjikian L, Abukhashabh A, Roure-Sobas Ch, Boibieux A, Denis $P$, et al. Diagnosis of Lyme-associated uveitis: value of serological testing in a tertiary centre. Br J Ophthalmol Published Online First: 28 June 2017.

34. Qureshi IA, Maldonado MF, Rodriguez GJ, Cruz-Flores S, Maud A. Optic neuritis in acute neuroborreliosis (Lyme disease) in the U.S.-Mexican border: Case report. World J Neuroscience. 2016:6:126-30.

35. Aberer E, Schwantzer G. Course of antibody response in Lyme borreliosis patients before and after therapy. ISRN Immunology 2012; 2012, Article ID 719821, 4 pages.

36. Lesser RL, Kornmehl EW, Pachner AR, Kattah J, Hedges TR, Newman NM, et al. Neuro-ophthalmologic manifestations of Lyme disease. Ophthalmology. 1990;97:699-706.

37. Dersch R, Hottenrott T, Schmidt S, Sommer H, Huppertz HI, Rauer S, et al. Efficacy and safety of pharmacological treatments for Lyme neuroborreliosis in children: a systematic review. BMC Neurology. 2016;16:189. 